\title{
Research on Intelligent Commercial Data Mining Model Based on Multi - Method Fusion in High - speed Optical Fiber Communication Network
}

\author{
Xiaoxuan Sun ${ }^{1}, \quad$ Xiaoming Zha ${ }^{2}$ \\ ${ }^{1}$ Yunnan Vocational College of Mechanical and Electrical Technology, Yunnan, Kunming, 650000 \\ ${ }^{2}$ ISoftStone information technology (Group ) Co., Ltd
}

Keywords: High - speed Optical Fiber Communication Network, Multi - Method Fusion, Intelligent Commercial, Data Mining, System Structure, Model Analysis

\begin{abstract}
In the context of the rapid development of Chinese economy and society, the commercial data has been greatly increased in the long run, so that the operation of the enterprise from the relevant data to find the corresponding decision support tools are getting more and more attention. The development of high-speed optical fiber communication network has further promoted the data mining and commercial functions in the operation and development of enterprises play a vital role. In the process of commercial transactions, the corresponding data contains a considerable part of the business decision-making process useful knowledge and content, and the application of data mining technology can be scientific and timely development of business operations hidden model and relationship problems. This paper discusses and analyzes the structure of general data mining system based on multi-method fusion in high-speed optical fiber communication network, the deficiency of common data mining system structure in commercial operation and the corresponding improvement ideas, the status quo of intelligent business data mining model, and Intelligent business data mining model of the future development trend of four important content, aimed at further strengthening the high-speed optical fiber communication network based on multi-method integration of intelligent business data mining model application and development, thus promoting our business stable and healthy operation.
\end{abstract}

\section{Introduction}

In the process of development and operation of our business is essential, there will be a lot of data, which contains the business process of running a variety of information and circumstances, enterprises in the actual operation and development of effective pediatrics in the decision-making process in the application of the corresponding data to a certain extent to the further development of enterprises to bring good economic benefits. It is of great practical significance to carry out the comprehensive excavation of the true meaning of the data in the operation of the enterprise. The concrete application of the data mining mode can effectively excavate the correlation between the data in the enterprise and help the corresponding staff make a decision-making work [1]. Data mining in the enterprise operation and development is not a simple technology or software, but a variety of professional and technical application of a comprehensive combination of data processing methods. 


\section{The Structure of High-Speed Optical Fiber Communication Network Based on Multi-Method Integration of the General Data Mining System}

The use of data mining tools in the course of business operations is to provide an enforceable solution based primarily on the resolution of specific issues in business. In the enterprise operation to design the corresponding data mining specific algorithm, the corresponding staff that must run the data characteristics and a certain mining requirements to fully consider the comprehensive operation of the enterprise has been generated by the actual data mining algorithm optimization [2]. Under normal circumstances, the commercial operation of the data mining model is comprehensive to the entire data warehouse and large relational database as the prerequisite basis, and have a certain data query, data analysis and other corresponding functions, is the enterprise decision-making process to support the system key components, the following figure shows the common characteristics of the data mining system.

\section{The Lack and the Corresponding Improvement Ideas of the General Data Mining System Structure in the Commercial Operation}

The Defect Analysis of the General Data Mining Model Structure. To a certain extent, GM's data mining model has been able to meet the needs of the broad masses of the people, but in the actual operation there are still corresponding problems and defects, the general performance of the following: the actual efficiency of data mining is not very Efficient, need to be further improved and strengthened; cannot be comprehensive and effective application of data mining historical model; different systems in the actual operation of each other is less operational; and in the face of different application objects do not have more high targeted [3].

The Investigative Approach to Actively Improving the Efficiency of Data Mining. First, the corresponding data on the development of commercial operation should be pre-processed and the noise data can be effectively removed, in strict accordance with the actual mining requirements of the corresponding data processing, so as to effectively reduce the commercial operation of mining data amount [4]. Second, for data mining and specific data analysis of the actual requirements, the corresponding staff within the enterprise should be timely design of more efficient data mining calculation method. Third, a reasonable increase in data mining model application hardware performance and the use of certain processing technology to improve the data mining speed.

\section{The Intelligent Business Data Mining}

At present, under the influence of the continuous development of the network environment, the daily increase in the amount of data began to increase. Algorithms and theories are maturing, and the progress of computer computing power makes data mining become the core technology of enterprise mining information.

In the network information technology and the continuous development of computer technology, in order to obtain a certain problem of key data is no longer nonsense, but for a wide range of data, the number of data, relying on manual summary report is still unable to Implementation, the kind of according to the specified model, a simple analysis of statistical methods cannot meet the needs of data analysis. Therefore, we can use a variety of statistical methods, intelligent analysis of large data software began to appear, which is the current international statistics on the use of universal methods, data mining technology technical support, market needs to be taken seriously. Data mining as a new field, in the reasoning, scope, definition and expectations of differences, mining 
knowledge and information are from a huge database to achieve and many researchers in the database system, machine learning as a major problem [5]. Many experts in the field have shown a strong interest in data mining, for example, in the information services industry has also appeared in the individual application in the Internet data warehouse, online service vitality. In addition, MDT, LBS, FAIS and TASA have been produced under the influence of industry-university cooperation, and many researchers are currently regarded as the main fields of database system and machine learning technology.

Under the influence of the continuous development and progress of information technology, the current environment of commercial data mining also changes, the speed and intensity of enterprise competition gradually increase at the same time, the market transactions also allow enterprises to deal with and save the amount of data in this environment, the focus of the enterprise from the previous data, the collection into an effective use of the database to obtain the appropriate information [6]. How can enterprises meet the development needs of external competition, you need to rely on data mining technology under the model construction.

The data mining technology model transforms the previous data into a business policy that can be implemented, including understanding the full picture of the customer, predicting future behavior, and creating more value [7]. Such as the establishment of customer churn prediction model, to avoid the phenomenon of mobile customers, the establishment of credit assessment models and risks to reduce the risk of business processes; segment the market to tap new business opportunities and other business decisions, and only the information into business profits to be able to achieve data storage, customer relationship management system has the investment benefits. Therefore, the use of data mining began to become broad, in addition to the digital assets can be converted into a type of business revenue, but also can be used to audit the benefits of other systems.

\section{The Future of Data Mining}

The popularity of the Internet so that people into the explosion of the Internet era, before the master of the information began to drown in the flood of the development of the times. In the future business data mining process, data warehousing as a major area, data mining tools can be obtained through the data warehouse more benefits [8]. Data warehouses as a collection of data, the data may sometimes exceed the number of units, and retailers, consumer marketers or service companies to monitor the number of millions of customer transactions from the situation, the data warehouse as a super tell the computer with special software Implementation, but also enterprises to achieve "real-time operations" of the basic equipment, the realization of this operation that the product portfolio to adjust the marketing program to adjust.

Modern enterprise development process needs to pay attention to data, but also through effective measures to enhance the use of relevant data, will be transformed into useful information to create greater value. The results show that the data processed by enterprises will increase every 5 years, many companies do not exist the lack of data problems, excessive data duplication is the need to solve the main problem, which allows enterprises in the management and use of data There are more problems, so the market needs is the relevant data into a reliable, reliable information system [9].

Enterprise Data Warehouse will enter the relevant data into the Data Mart system, which will enter the data into a personal box, that is, personal terminals, including the use of company power play or other tools, which requires a complete system of database management tools can support the database hierarchy. Mr. Simon Dennis believes that this toolbox has a large Data Mining architecture, for small enterprises operating system, the smaller architecture can be in the small 
Mart operation [10].

Many business people will be put into the business intelligence to explore the process, the experts and scholars believe that the use of tools to analyze and detect data mining results will gradually become common. This kind of forecasting becomes practical before data dissemination requires more effort, modern enterprise body needs to collect a lot of data, including customers, markets, suppliers, future trends, competitors and other information [11]. However, the information overload and unstructured let the enterprise decision-making behavior misuse and confusion, therefore, the proper use of data mining technology, you can from the huge library of different knowledge and information as a decision-making, so that enterprises have more competitive advantage.

\section{Conclusion}

In summary, this paper focuses on the structure of general data mining system based on multi-method fusion in high-speed optical fiber communication network, the deficiency of common data mining system structure in commercial operation and the corresponding improvement ideas, the status quo of intelligent business data mining model and the future development trend of intelligent business data mining model. It is hoped that we can further strengthen the application and development of intelligent business data mining model based on multi-method fusion in high-speed optical fiber communication network, so as to promote the commercial Smooth and healthy operation

\section{References}

[1] Han Ruyi, Li Tao, Zhou Qifeng, Huang Yue, Zheng Li. Intelligent Inventory Management System iMiner [J]. Journal of Xiamen University (Natural Science Edition), 1-13.

[2] Huang Xiaobin, Zhang Xingwang.Study and prospect of network dynamic data mining [J / OL]. Library and Information Work, 2015 (10)

Http: //www.cnki.net/kcms/detail/10.13266/j.issn .0252-3116.2015.10.001.html

[3]. Mining large data era business model - millet technology company chairman and CEO Lei Jun in 2015 Guiyang International Big Data Industry Expo speech[J]. Information technology and information, 2015, 05: 47

[4] Zhao Xiangmo, Hui Fei, Shi Xin, Ma Junyan, Yang Lan. Pan-traffic information service system concept, architecture and key technology[J]. Journal of Traffic and Transportation Engineering, 2014, 04: 105-115.

[5] Yuan Hongchun, Xiong Fanlun, Huai Xiaoyong.Spatial Data Mining and Its Integration Framework with Intelligent System [J]. Information and Control, 2002, 04: 304-309.

[6] YANG Shan-sheng, LU Wen-cong, GU Tian-hong, LU Zhi-rong, LIU Xin, YANG Ming.Application of optimization and monitoring system for synthetic ammonia process based on data mining [J]. Chemical Automation and Instrumentation, 2010, 07: 76-78.

Design and Implementation of Distributed Intelligent Intrusion Detection System[J]. Journal of Xi'an University of Technology, 2008, 02: 228-232. 
[7] Liu Tao, Bai Liang, Hou Yuobin. Design and Implementation of Distributed Intelligent Intrusion Detection System Model[J]. Journal of Xi'an University of Technology, 2008, 02: 228-232.

[8] Guo Weigang, Song Yibing, Luo Yiling. User access pattern mining of e-commerce website [J]. Microelectronics and Computer, 2005, 05: 170-174.

[9]. The 8th China Data Mining and Business Intelligence Seminar and Cross-Strait Application Statistics Seminar [J]. Economics and Management Research, 2011, 09: 129.

[10]T.V. Chabata,D. Kiboi Boiyo,E.K. Rotich Kipnoo,R.R.G. Gamatham,A.W.R. Leitch,T.B. Gibbon. Signal Monitoring and Performance Stability Evaluation Tool in a High Speed Optical Communication Network[J]. Optik - International Journal for Light and Electron Optics, 2016

[11]Abd El Naser A. Mohammed, Mohamed A. Metawe'e, Ahmed Nabih Zaki Rashed, and Mahmoud M. A. Eid. Distributed Optical Raman Amplifiers in Ultra High Speed Long Haul Transmission Optical Fiber Telecommunication Networks[J]. International Journal of Computer and Network Security, 2009, 11 